- to provide emotional support to the dying and their families

- to facilitate increased understanding about the emotional and social needs of the dying, and their families, within the community and across the hospital environment.

Methods On the whole, healthcare professionals refer patients/ families to the service manager who then meets the patients/families to understand their needs. An appropriate "companion" is allocated by the service manager and the "companion" is then introduced to the patient/family to provide face-to-face support tailored to their needs.

The service is evaluated on a continuous basis, using:

- Stakeholder feedback: from patients/families, staff and "companions" to explore the impact of the service on improving emotional support

- Activity data: completed by the "companions" capturing what support was delivered.

Results An earlier review suggested that hospital staff, dying patients and their families had very positive experiences of the service, with nursing staff seeing benefits in terms of patient and family wellbeing and their own stress levels.

The initial pilot has increased from three adult wards to supporting all 20, and has received over 250 referrals since launch in August 2014.

The service is the only one of its kind in the country, and was outlined in the Care Quality Commission report as an example of outstanding practice.

\section{P-173 THE HOSPICE FRIENDLY HOSPITALS PROGRAMME IN IRELAND}

Grace O'Sullivan, Marie Lynch. Irish Hospice Foundation, Dublin, Ireland

\subsection{6/bmjspcare-2016-001245.195}

Background Almost 30,000 people die in Ireland each year. 43\% of people in Ireland die in acute hospitals; that's around 35 people every day. The Irish Hospice Foundation identified a deficit in the care of patients and their families at the end of life and in 2007 established the Hospice Friendly Hospitals (HFH) Programme in partnership with Ireland's National Health Service Executive. The programme was established in order to introduce hospice principles into hospital practice

Aim The HFH Programme seeks to ensure that end of life, palliative and bereavement care is central to the everyday business of Irish hospitals.

Methods The HFH Programme designed a suite of quality improvement interventions for hospital staff. The programme

- Developed the Quality Standards for End of Life Care and a suite of guidance documents for hospital staff.

- Improved the culture of end-of-life care through education initiatives, a national nurse practice development programme and promotion of symbolic resources.

- Influenced the health system to raise the profile of end of life care by establishing.

- End-of-Life Care Coordinator posts.

- End-of-Life Care/Bereavement Care Committees in hospitals.

- Three national leadership networks for hospital staff.
Results

- Over 40 public and private hospitals are linked to the HFH Programme. This represents over 90\% of public hospitals in Ireland.

- Palliative, end-of-life and bereavement care is a core component of hospital operational plans.

- Hospitals have established a governance structure (committee) to oversee quality improvement projects.

- Symbolic resources e.g. the end-of-life symbol are used widely.

- Over 20 refurbishment projects complete e.g. mortuaries, family rooms, bereavement suites through the Design \& Dignity Grants Scheme.

- The Quality Standards for End of Life Care underpin new national standards for bereavement care in maternity settings.

- The HFH Programme is working with organisations in Northern Ireland, the UK and Canada to disseminate learning.

\section{P-174 TRANSFORMING END OF LIFE CARE IN THE COMMUNITY AND ACUTE HOSPITAL}

${ }^{1}$ Fiona Tucker, ${ }^{1}$ Lynn Kelly, ${ }^{1,2}$ Claire Capewell, ${ }^{2}$ Linda Dewhurst. 'St Catherine's Hospice, Preston, UK; ${ }^{2}$ LTHTR

10.1136/bmjspcare-2016-001245.196

Background The Transforming End of Life Care in the Community Programme was based on the success of the Transform Acute Hospitals programme. Both programmes aim to promote multiprofessional team communication and to improve end of life care. Two local Clinical Commissioning Groups provided funding for the community programme, which launched in April 2015, following introduction of the programme to health-professionals by means of masterclasses.

Aim The hospital programme aims to accommodate patients going back to their preferred place of care/death. The community programme aims to keep people in their preferred place of care/death. It intends to promote the use of supportive care registers and improve the incidence, quality and recording of future wishes, preferences, wishes and values - Advance Care Planning (ACP).

Jointly, both programmes are facilitating implementation of the new Electronic Palliative Care Co-ordination System.

Methods The hospital programme utilises teaching time with hospital staff. In the community, Protected Education Training time in GP practices is used, incorporating district nursing teams.

Training covers ACP, DNACPR and end-of-life care, and has extended to community therapy teams and nursing/care homes, with stand-alone sessions for NWAS and PTS. It incorporates the 'One Chance to Get it Right' and the 'NICE Guidance for the Care of the Dying Adult' documents. The education is measured with both pre and post impact and evaluation.

Results The number of people trained as of $31 / 03 / 16$ is 628 . Completed figures from cohort one indicate a $56 \%$ increase in the number of people identified as being in their last year of life. Records of ACP and DNACPR discussions have increased by almost 21\%.

In 2008, average local DiUPR figures were 33.8\% (England $37.8 \%$ ). In 2014 , these figures were $39.2 \%$ and $44.7 \%$ respectively. We wait to see whether this education initiative, in combination with other aspects of local strategy will lead to a further improvement in DiUPR. 